\title{
DISTRIBUTED BEAM LOSS MONITOR BASED ON THE CHERENKOV EFFECT IN OPTICAL FIBER
}

\author{
Yu.I. Maltseva*, V.G. Prisekin, BINP SB RAS, Novosibirsk, Russia \\ F.A. Emanov, BINP SB RAS and NSU, Novosibirsk, Russia \\ A.V. Petrenko, BINP SB RAS, Novosibirsk, Russia; CERN, Geneva, Switzerland
}

\section{Abstract}

A distributed beam loss monitor based on the Cherenkov effect in optical fiber has been implemented for the VEPP-5 electron and positron linacs and the $510 \mathrm{MeV}$ damping ring at the Budker INP. The monitor operation is based on detection of the Cherenkov radiation generated in optical fiber by means of relativistic particles created in electromagnetic shower after highly relativistic beam particles (electrons or positrons) hit the vacuum pipe. The main advantage of the distributed monitor compared to local ones is that a long optical fiber section can be used instead of a large number of local beam loss monitors. In our experiments the Cherenkov light was detected by photomultiplier tube (PMT). Timing of PMT signal gives the location of the beam loss. In the experiment with $20 \mathrm{~m}$ long optical fiber we achieved $3 \mathrm{~m}$ spatial resolution. To improve spatial resolution optimization and selection process of optical fiber and PMT are needed and according to our theoretical estimations $0.5 \mathrm{~m}$ spatial resolution can be achieved. We also suggest similar techniques for detection of electron (or positron) losses due to Touschek effect in storage rings.

\section{INTRODUCTION}

VEPP-5 Injection Complex [1] now is under commission and will supply BINP RAS colliders with electron and positron beams. The VEPP-5 Injection Complex consists of $270 \mathrm{MeV}$ driving electron linac, $510 \mathrm{MeV}$ positron linac and dumping ring. In order to control beam losses along beamline during the Complex commissioning and operation we proposed to use a distributed beam loss monitor based on the Cherenkov effect in optical fiber.

This type of beam loss monitor has been developed at several facilities such as FLASH (DESY), SPring-8 (RIKEN/JASRI), CLIC Test Facility (CERN) [2-4]. The monitor overview is given by T. Obina [5].

Compared with other distributed beam loss monitors such as long ionization chamber and scintillating fiber, optical fiber beam loss monitor (OFBLM) has the following advantages: fast response time $(<1 \mathrm{~ns})$ which allows to detect multi-turn beam losses in a storage ring, near zero sensitivity to background signal (mainly gamma radiation) and synchrotron radiation, unlike scintillating fiber. Moreover, optical fiber is insensitive to magnetic field, but it is susceptible to radiation damage (except quartz fiber), which limits fiber lifetime. Another disadvantage of the OFBLM is an issue with its calibration.

Yu.I.Maltseva@inp.nsk.su

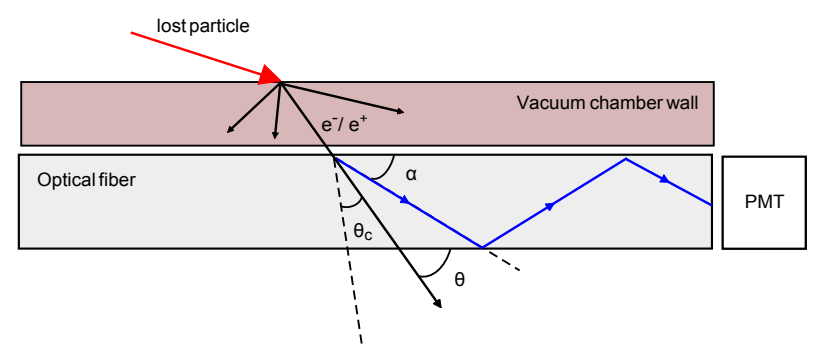

Figure 1: Scheme of beam loss monitor.

\section{PRINCIPLE OF BEAM LOSS MONITOR}

The basic idea behind the OFBLM is to detect a burst of the Cherenkov radiation (CR) generated in optical fiber by means of relativistic particles created in electromagnetic shower after highly relativistic beam particles (electrons or positrons) hit the vacuum pipe. Some of the Cherenkov photons propagate through the fiber and can be detected by PMT (Fig. 1). The following physical processes determine the beam loss detection principle of the OFBLM.

\section{Cherenkov Radiation}

The Cherenkov radiation is an electromagnetic radiation emitted in a cone around the moving charged particle with cone semi-angle $\theta_{c}$ :

$$
\cos \theta_{c}(\lambda)=\frac{1}{\beta n(\lambda)}
$$

where $\lambda$ - radiation wavelength, $\beta$ - particle velocity $(\beta=v / c), n$-medium refractive index. Neglecting refractive index dispersion, the number of the Cherenkov photons from a single electron or positron per unit photon energy per unit path length of the particle in a medium is given by:

$$
\frac{d N}{d \lambda d x}=\frac{2 \pi \alpha \sin ^{2} \theta_{c}}{\lambda^{2}}
$$

where $\alpha$ is the fine structure constant. According to eq. (2), the greater part of the Cherenkov photons is emitted in the UV range of the spectrum. For relativistic electron passing through plastic optical fiber $(n=1.492)$ the CR in visible spectrum $(400 \mathrm{~nm}<\lambda<700 \mathrm{~nm})$ is generated with 30 photons per mm and emission semi-angle $\theta_{c}$ is about $48^{\circ}$.

Besides the $\mathrm{CR}$, optical transition radiation can be detected by PMT. However, the number of optical transition radiation photons was estimated to be $10^{4}$ times less than the number of the $\mathrm{CR}$ photons and thus can be neglected. 


\section{Signal Propagation}

The emitted Cherenkov radiation propagates through optical fiber by means of total internal reflection. This is the case when light (see Fig. 1) propagates with angles $\alpha$ relative to the fiber axis lower than:

$$
\alpha \leq \alpha_{\max }=\sin ^{-1}\left(N A / n_{c o}\right)
$$

where $N A$ - optical fiber numerical aperture, $n_{c o}-$ refractive index of optical fiber core. For example, for optical fiber with $N A=0.47$ and $n_{c o}=1.492, \alpha \leq 18^{\circ}$.

Neglecting attenuation effects, the probability for the CR to be transmitted in the fiber by guided rays is given by [6]:

$$
P=\frac{1}{\pi} \cos ^{-1}\left(\frac{\beta n_{c o} \cos \alpha_{\max }-\cos \theta}{\sin \theta \sqrt{\beta^{2} n_{c o}^{2}-1}}\right)
$$

where $\theta$ - angle of the charged particle propagation relative to the fiber axis.

\section{Electromagnetic Shower}

For electromagnetic shower simulation G4beamline code [7] was used. Angular distribution of secondary electrons and positrons relative to beam direction was obtained (Fig. 2).

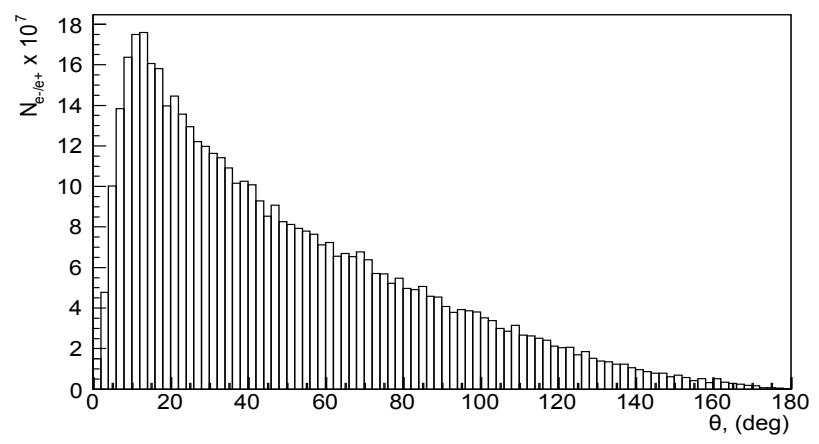

Figure 2: Angular distribution of secondary e-/e+ passing through optical fiber relative to beam direction. $270 \mathrm{MeV}$ electron beam with $10^{10}$ electrons hits $2 \mathrm{~mm}$ steel vacuum pipe with $1^{\circ}$ incident angle. Fiber is placed at the loss point. Total number of charged particles is $0.5 \cdot 10^{10}$.

According to eq. (4), secondary charged particles passing through optical fiber with angle $\theta$ between $30^{\circ}-66^{\circ}$ and $114^{\circ}-150^{\circ}$ relative to the fiber axis generate the Cherenkov radiation which is trapped in optical fiber and transmitted by guided rays. This results in trapping of about $33 \%$ and $5 \%$ of generated radiation, respectively. Consequently, downstream signal sensitivity is 7 times higher than upstream one.

For the CR to be generated kinetic energy of lost particles must be greater than $10 \mathrm{MeV}$ for electron (or positron) beam and greater than $5 \mathrm{GeV}$ for proton beam.

\section{MONITOR SPATIAL RESOLUTION OPTIMIZATION}

Monitor spatial resolution depends on light dispersion in optical fiber, PMT and electronics resolution. There are two main dispersion types in fiber: modal and chromatic. Transmitting signal through optical fiber of length $L$ each dispersion contribution to pulse broadening can be estimated as:

$$
\begin{aligned}
& t_{\text {mod }} / L=1 / c n_{c o}\left(1 / \cos \alpha_{\text {max }}-1\right), \\
& t_{c h r} / L \approx 1 / c\left(n_{c o}\left(\lambda_{1}\right)-n_{c o}\left(\lambda_{2}\right)\right) .
\end{aligned}
$$

where $\lambda_{1}$ and $\lambda_{2}$ determine spectral range.

In multimode optical fiber with step-index profile signal lengthening is effected mainly by modal dispersion. In multimode fiber with graded-index profile chromatic dispersion is dominant and modal one is negligible. In singlemode fiber there is no modal dispersion, but its lower pulse distortion comes at the expense of lower signal sensitivity. For step-index multimode fiber which was used in our tests $t_{\text {mod }} / L=0.25 \mathrm{~ns} / \mathrm{m}, t_{c h r} / L=0.05 \mathrm{~ns} / \mathrm{m}$, i.e. modal dispersion contributes 5 times greater to monitor spatial resolution then chromatic one.

PMT signal strength and FWHM of the signal as a function of the fiber length were also studied (Fig. 3). For the VEPP-5 Injection Complex, taking into consideration strength of the light signal and its width, optimal length of the fiber is about $20 \mathrm{~m}$.

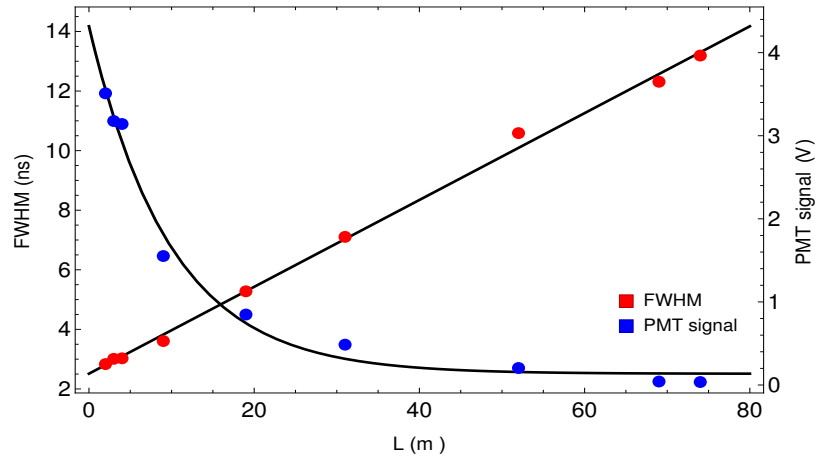

Figure 3: PMT signal (blue) and FWHM of the signal (red) as a function of the fiber length.

To improve monitor spatial resolution graded-index multimode optical fiber, singlemode one or step-index multimode fiber along with collimator should be used as well as PMT and electronics with small time resolution. Micro-channel plate PMT is a good candidate. Dependence of the monitor spatial resolution on length of the collimator attached to the end of the fiber was studied. For $10 \mathrm{~m}$ long step-index multimode fiber collimator can improve monitor spatial resolution by up to $20 \%$.

The Cherenkov signal can be detected at either downstream or upstream end of the fiber. In case of multiple losses originated from successive magnet elements 5 times better spatial discrimination between losses can be achieved by signal detection at the upstream end of the fiber compared 

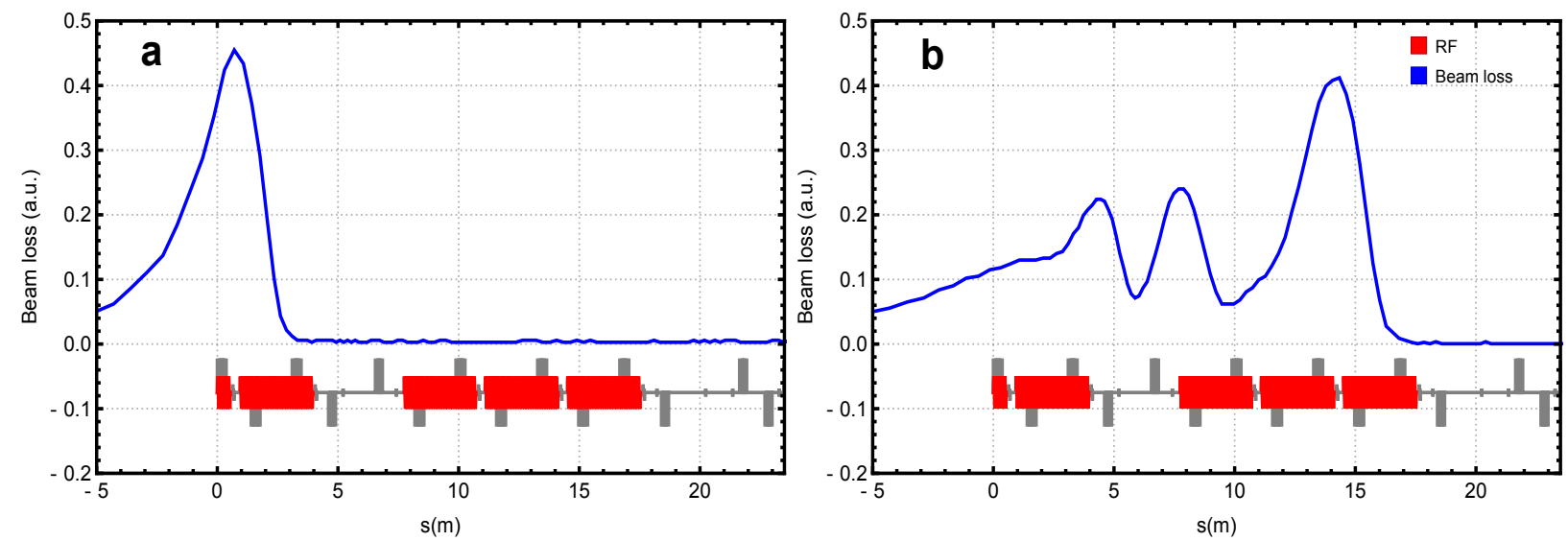

Figure 4: Beam losses: a) beam was dumped at the end of the 1st RF-structure, $20 \mathrm{~m}$ fiber was used; b) at the end of the 4th RF-structure, $60 \mathrm{~m}$ fiber was used.

with the downstream one. This is due to the fact that beam velocity $\beta c$ is greater than speed of light in optical fiber $c / n$. Despite upstream signal sensitivity is 7 times lower than downstream one, the former is preferable.

\section{EXPERIMENTAL RESULTS}

A prototype of the OFBLM was made using $20 \mathrm{~m}$ and $60 \mathrm{~m}$ long plastic step-index multimode fibers Avago Tech. HFBR-RUS500Z ( $1 \mathrm{~mm}$ core diameter, $n_{c o}=1.492$ and $N A=0.47)$. To detect the CR signal at the downstream end of the fiber FEU-85 PMT (300-600 nm spectral sensitivity range) was used. The prototype of the OFBLM was installed in the VEPP-5 electron linac and tested in a controlled manner by dumping beam with dipole correctors. The experimental results are shown in Fig. 4.

As one can see from Fig. 4a FWHM of the signal approximately corresponds to 3 meters for $20 \mathrm{~m}$ long optical fiber and $1.5 \mathrm{~m}$ beam length. For single bunch $(4 \mathrm{~mm})$, collimator attached to the end of the fiber or graded-index multimode optical fiber or singlemode one and micro-channel PMT will allow us to achieve $0.5 \mathrm{~m}$ monitor spatial resolution limited mostly by ADC characteristics. In Fig. $4 \mathrm{~b}$ for $60 \mathrm{~m}$ long optical fiber besides controlled beam loss at the end of the 4th RF-structure one can see beam losses in multiple locations along linac.

\section{TOUSCHEK EFFECT}

In storage rings besides irregular beam losses there are beam lifetime losses due to Touschek effect (loss mechanism driven by large-angle Coulomb scattering within the high charge bunch). This effect results in losses of scattered particles with energy variation exceeding the energy acceptance of the ring. For the VEPP-5 dumping ring with $28 \mathrm{~m}$ circumference and $2 \cdot 10^{10}$ electron bunch charge the beam lifetime is roughly $10 \mathrm{~min}$ and the average number of lost electrons per turn is 2. According to our theoretical estimations, the OFBLM can detect a single electron (or positron) hitting the vacuum pipe with probability about $1 \%$ for the VEPP-5 beam parameters. Hence, using two optical fibers placed on both sides of vacuum chamber the monitor can detect $10^{8}$ events. It's sufficient to obtain precise distribution of the beam losses due to Touschek effect in a storage ring.

\section{CONCLUSION}

The distributed beam loss monitor based on the Cherenkov effect in optical fiber has been tested in the VEPP-5 electron linac. $3 \mathrm{~m}$ monitor spatial resolution for $20 \mathrm{~m}$ long optical fiber was obtained. Using step-index multimode fiber along with collimator or graded-index multimode optical fiber or singlemode one and micro-channel plate PMT $0.5 \mathrm{~m}$ spatial resolution can be achieved. To achieve better spatial discrimination between successive losses one should detect the light signal at the upstream end of the fiber. The monitor ability to detect single particle losses and high signal-to-noise ratio make it possible to use the monitor for detection of electron (or positron) losses due to Touschek effect in storage rings.

\section{REFERENCES}

[1] K.V. Astrelina et al. "Production of Intense Positron Beams at the VEPP-5 Injection Complex", Journal of Experimental and Theoretical Physics, Vol. 106, Issue 1, p. 77 (2008).

[2] F. Wulf, M. Körfer, "Beam Loss and Beam Profile Monitoring with Optical Fibers", Proc. DIPAC09, Basel, Switzerland (2009) 411.

[3] X.-M. Maréchal et al. "Beam Based Development of a Fiber Beam Loss Monitor for the Spring-8/XFEL", Proc. DIPAC09, Basel, Switzerland (2009) 234.

[4] S. Mallows et al. "Fiber Based BLM System Research and Development at CERN", Proc. HB2012, Beijing, China (2012) 596.

[5] T. Obina, "Optical Fiber Based Loss Monitor for Electron Storage Ring”, Proc. of IBIC2013, Oxford, UK (2013) 638

[6] S.H. Law, S.C. Fleming, N. Suchowerska, D.R. Mckenzie, Appl, Opt. 45 (2006) 9151.

[7] http://www . muonsinternal . com/muons3/G4beamline 